\title{
Boom-Constrained Drag Minimization for Design of Supersonic Concepts
}

\author{
Sriram K. Rallabhandi* \\ National Institute of Aerospace, Hampton, VA 23666 \\ $\mathrm{Wu} \mathrm{Li}^{\dagger}$ \\ NASA Langley Research Center, Hampton, VA 23681 \\ Karl Geiselhart $\ddagger$ \\ NASA Langley Research Center, Hampton, VA 23681
}

\begin{abstract}
This paper presents an approach to modifying an existing baseline configuration that has been designed to achieve low-boom characteristics in order to minimize drag while not severely penalizing baseline sonic boom levels. The baseline configuration that was used is the result of using a mixed-fidelity CFD-based low-boom design process that has been tested and verified. Shape modifications are carried out by using arbitrary shape-deformation algorithms. The focus of this paper is the integration of several key enabling techniques and methods for efficient redesign under stringent constraints.
\end{abstract}

\section{Nomenclature}

Symbols

$\begin{array}{ll}C_{D} & \text { Drag coefficient. } \\ C_{L} & \text { Lift coefficient. } \\ O P R & \text { Over-pressure ratio. } \\ P L d B & \text { Perceived loudness in decibels. } \\ w_{d} & \text { Weighting factor for drag coefficient. } \\ w_{p} & \text { Weighting factor for PLdB. } \\ X_{i} & \text { Design variables. }\end{array}$

\section{Introduction and Motivation}

$\mathrm{R}_{\mathrm{s}}^{\mathrm{s}}$ ESEARCHERS at NASA Langley Research Center and elsewhere are dedicated to overcoming the challenges that are associated with overland supersonic flight. The failure of any existing aircraft to overcome the problems that are associated with this type of flight is indicative of the difficulties that are inherent in the practical design of aircraft to mitigate sonic boom phenomenon.

The importance of aircraft design for sonic boom mitigation has been well-known in the supersonics design community for more than four decades. Several previous studies have discussed the analyses that are needed for sonic boom prediction. ${ }^{1,2}$ In addition to prediction, sonic boom minimization theory has been around since the 1960s. Originally developed by Seebass and George, ${ }^{3}$ boom minimization theory has been extended into hybrid forms but essentially maintains the linearized concepts of weak shocks that have been laid out by Whitham ${ }^{4}$ and others. ${ }^{5-7}$ Aircraft design for sonic boom minimization requires matching the near-field pressure distributions to those prescribed by boom minimization theory while not significantly degrading the performance in other aspects. Most often, supersonic aircraft design studies

${ }^{*}$ Research Scientist II, National Institute of Aerospace.

${ }^{\dagger}$ Senior Research Engineer, Aeronautics Systems Analysis Branch, NASA Langley Research Center.

${ }^{\ddagger}$ Aerospace Engineer, Aeronautics Systems Analysis Branch NASA Langley Research Center. 
have focused more on sonic boom than other performance measures simply for the reason that boom mitigation is one of the most stringent requirements for overland supersonic flight. Previous work has looked at creating suitable target equivalent-area distributions that produce front- and aft-shaped sonic boom ground signatures ${ }^{8}$ as well as generate a baseline aircraft that closely follows the desired target equivalent-area distribution. In this paper, the focus is on reshaping the baseline in a smooth manner in order to reduce drag without degrading sonic boom characteristics.

\section{Background}

Before shape optimization can be attemped, an efficient low-boom baseline configuration is needed. In addition, a design process is needed that can change the aircraft shapes with the desired control and accuracy that is required for performing high-fidelity computational fluid dynamics (CFD) analyses. These are briefly described in the following sections.

\section{A. Baseline}

A multifidelity shaping process has been created, wherein depending on the desired level of fidelity, lowfidelity or high-fidelity (CFD) analyses are automatically linked to the shaping process. In addition, a mixed-fidelity mode is available; this uses a scheme in which the total CFD equivalent area is calculated once and the gap between the CFD equivalent-area and the target equivalent-area is reduced by smooth reshaping of the fuselage equivalent-area with the use of low-fidelity analysis. This smooth fuselage reshaping procedure is well documented ${ }^{9}$ as is the automation and integration of CFD in the aircraft design process. ${ }^{10}$

This process, shown in figure 1 , was developed ${ }^{11}$ to design and evaluate low-boom configurations with sufficient fidelity to determine that the calculated ground signature is reasonably representative of that particular configuration. The design portion is accomplished initially by using low-fidelity methods to achieve a low-boom signature, primarily by reshaping the fuselage. Further refinement of that configuration and computation of the corresponding signature is accomplished with the use of both the CFD-generated lift and the equivalent-area that is attributable to volume from the CFD watertight geometry. A mixed-fidelity mode ${ }^{11}$ is run as described in the previous paragraph until a satisfactory result is obtained. With the use of classical ${ }^{3}$ sonic boom target equivalent-area distributions, the fuselage is smoothly shaped by carrying four iterations through the mixed-fidelity shaping process to obtain a baseline configuration as shown in figure 2. An off-body pressure distribution of that configuration can then be calculated by using CFD with a stretched grid $\left(\right.$ SSGRID $\left.^{12}\right)$ and that signature can be propagated to the ground by using PCBOOM $4^{13}$ to verify the mixed-fidelity design result. In this study however, stretched-grid computations are avoided in the interest of limiting the required computational time.

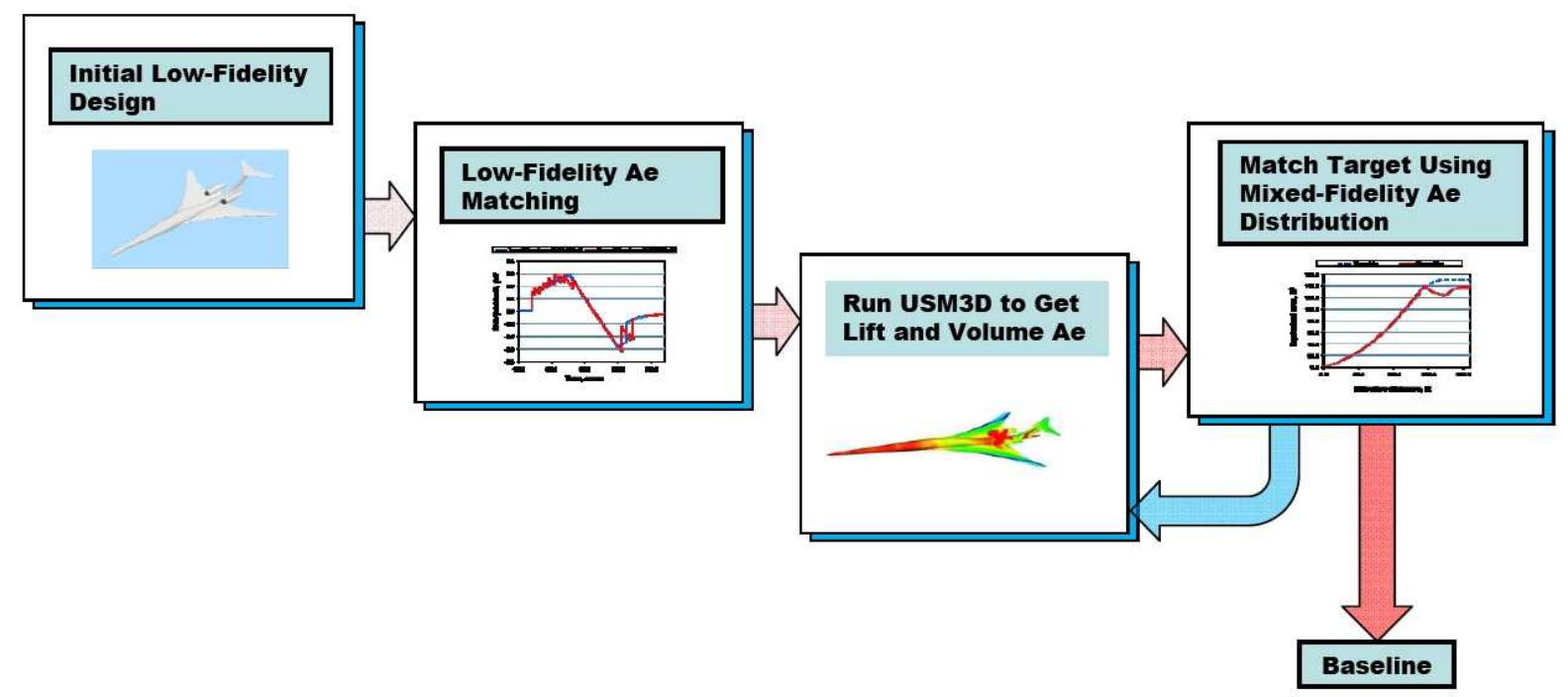

Figure 1. Baseline generation process.

This baseline concept is assumed to have a takeoff weight of 100,000 lbs and aircraft length of $170 \mathrm{ft}$. and is designed for a cruise Mach number of 1.8. Various analysis codes with varying levels of fidelity are used to evaluate the performance in different disciplines, and the interested reader should refer to other 


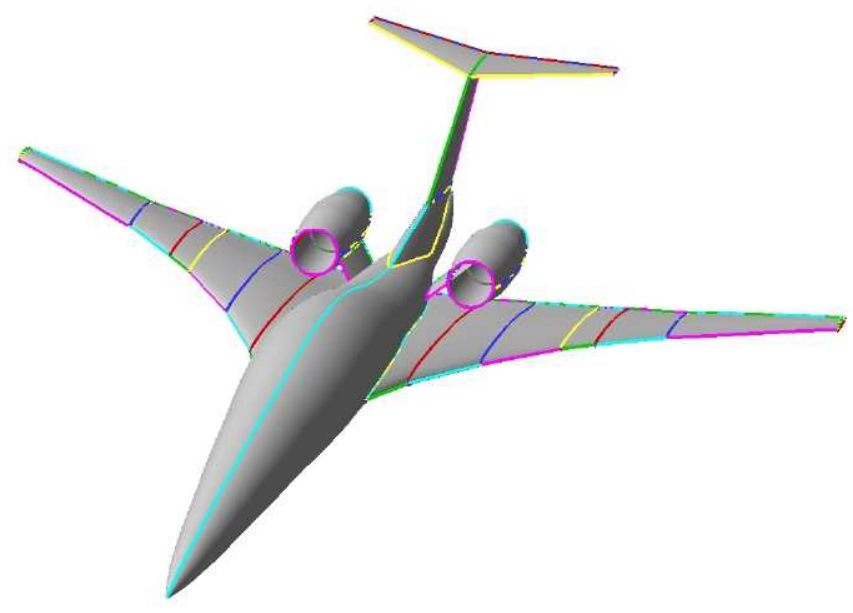

Figure 2. Baseline concept.

studies $^{9-11}$ for more information.

\section{B. Free-Form Shape Deformation}

Free-Form shape deformation is a technique to smoothly vary a given geometry according to a set of control parameters. The idea is to create a parametric volume around a region to be deformed. Once this volume is set, any deformation of the volume changes the underlying geometry. A tool called SCULPTOR $^{14}$ was used in this study. Additional details on the shape parameterization and deformation are given in the next section.

\section{Optimization Approach}

This paper demonstrates an approach to integrate the SCULPTOR tool with CFD analysis to result in an automated process for shape optimization. This integration process represents a multifidelity lowboom and low-drag design capability. The optimization process is shown in figure 3 ; the most relevant parameters $\left(X_{i}, C_{L}, C_{D}, P L d B\right.$ and $\left.O P R\right)$ are passed to the optimizer. The CFD analysis that is used in this study is USM3D, ${ }^{15,16}$ an unstructured tetrahedral mesh solver.

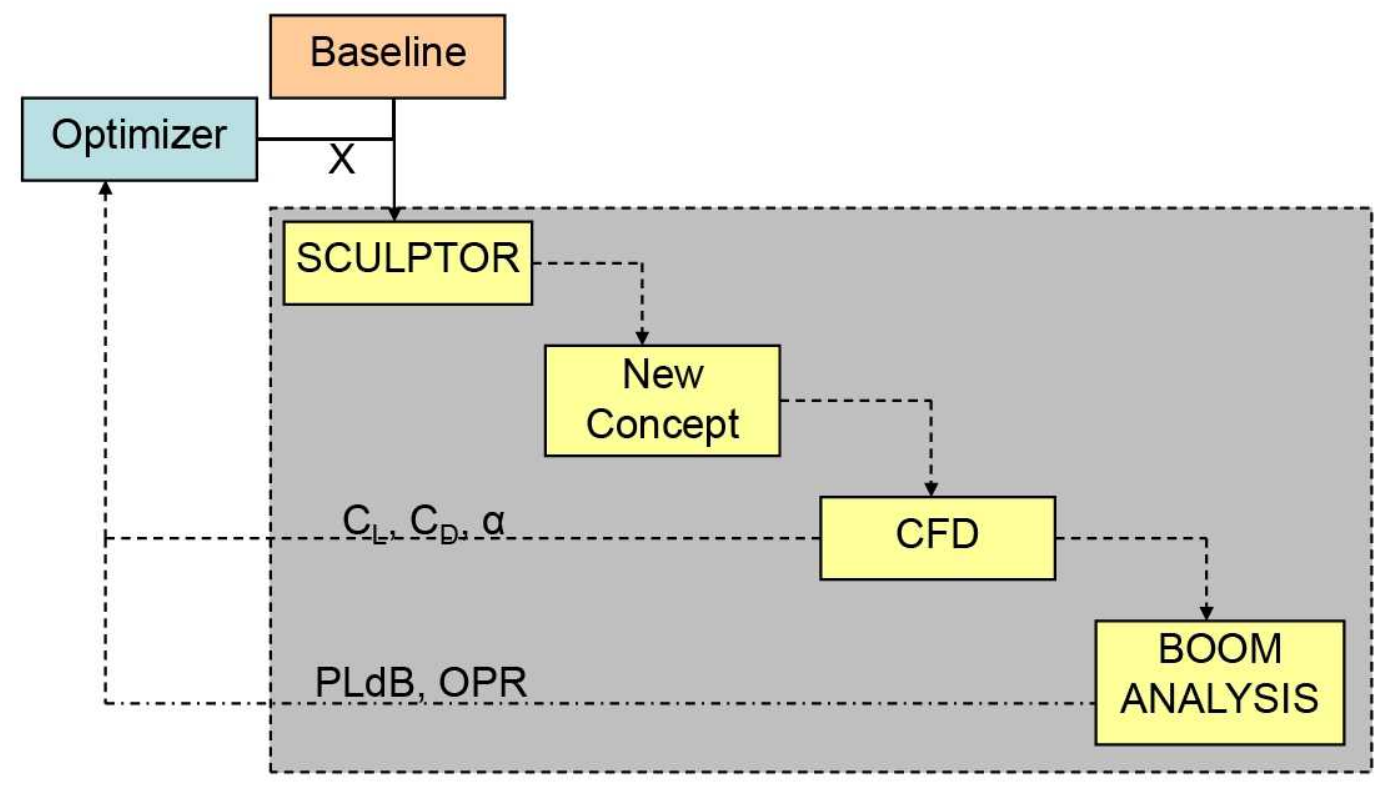

Figure 3. Flowchart of the optimization problem.

The optimization problem of finding the best deformed shape can be formulated as a boom-constrained drag-minimization problem (see Eq. 1). If the calculated perceived loudness (PLdB) of the ground 
signature is greater than the baseline value, then a penalty term is added to the weighted drag coefficient. The drag weighting constant $w_{d}$ is given a value of 1000.0 to convert the actual drag coefficient to drag counts and the PLdB weighting constant $w_{p}$ is given a value of 1 to allow one drag count tradeoff with $1 \mathrm{PLdB}$. As the aircraft shape changes based on changes in the design variables $X_{i}$, the lift coefficient remains equal to that of the baseline because the angle of attack is permitted to vary. The optimizer that is used in this study is Design Explorer ${ }^{17}$ which comes packaged with ModelCenter. ${ }^{18}$ For each case in the optimization run, Design Explorer first runs an orthogonal array of design-variable points within the possible ranges to generate a kriging-based surrogate model for its internal computations and faster search steps.

$$
O b j= \begin{cases}\min _{X_{i}} w_{d} * C_{D}\left(X_{i}\right) & \text { If } P L d B \leq P L d B_{\text {baseline }} \\ \min _{X_{i}} w_{d} C_{D}\left(X_{i}\right)+w_{p}\left(P L d B-P L d B_{\text {baseline }}\right)^{2} & \text { If } P L d B>P L d B_{\text {baseline }}\end{cases}
$$

Many shape-optimization studies start off with a poor baseline, show improvement in the final results with respect to the baseline, and proclaim success for the underlying methodology. This is not the case here, as the baseline used in this work is the result of a detailed and careful multifidelity analysis and design process to minimize the sonic boom footprint. Therefore, in this case boom-constrained drag minimization is much more challenging.

The focus of this paper is wing reshaping. Figure 4(a) shows the control points over the wing of the baseline. The yellow highlighted points represent the movable points of the grid for shape deformation; the other points are the fixed control points. Nine parallel planes are located along the wing span at various spanwise locations. According to the cubic B-spline philosophy that is incorporated within the arbitrary shape-deformation engine of Sculptor, each control-point movement causes the smooth movement of two adjoining points in each of the six directions in three dimensional space. Beyond those two points, the control-point movement does not change the underlying mesh. Thus, the shape changes are smooth and localized. The region where the mesh is modified is depicted in figure 4(b). This region is called the arbitrary shape deformation (ASD) volume. The volume mesh outside this region remains the same as that of the baseline; hence rather than starting from the ambient conditions, using the CFD restart option from the baseline solution saves time because it provides a more accurate initial guess of the flow field. In order to isolate the volume mesh around the wing and provide a smooth transition at the boundaries of the ASD volume, the control points that are chosen for deformation are padded with two rows of points in all directions, as shown in Figure 4(a).

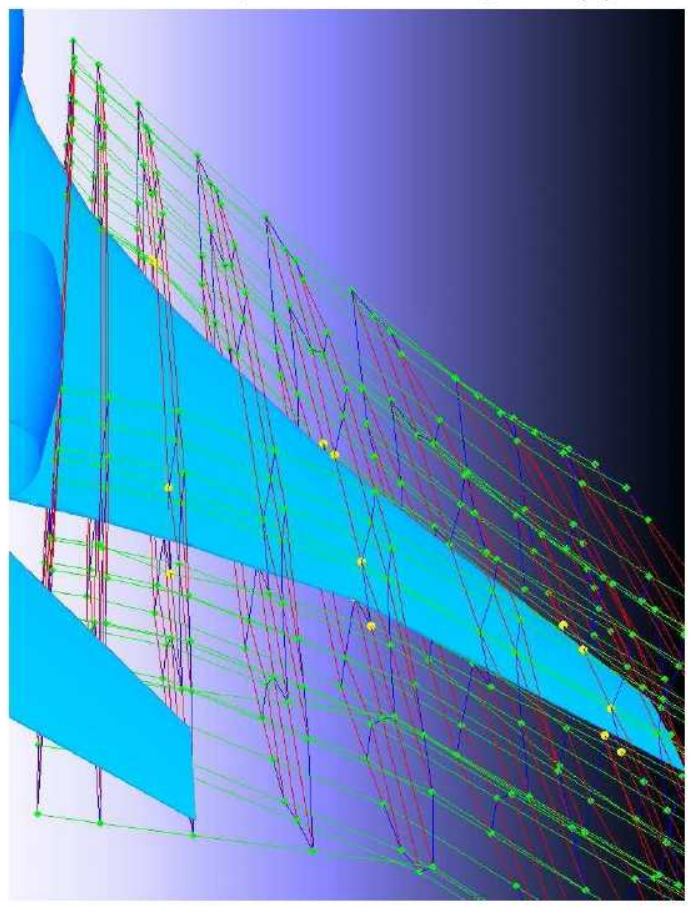

(a) Sculptor wing control points.

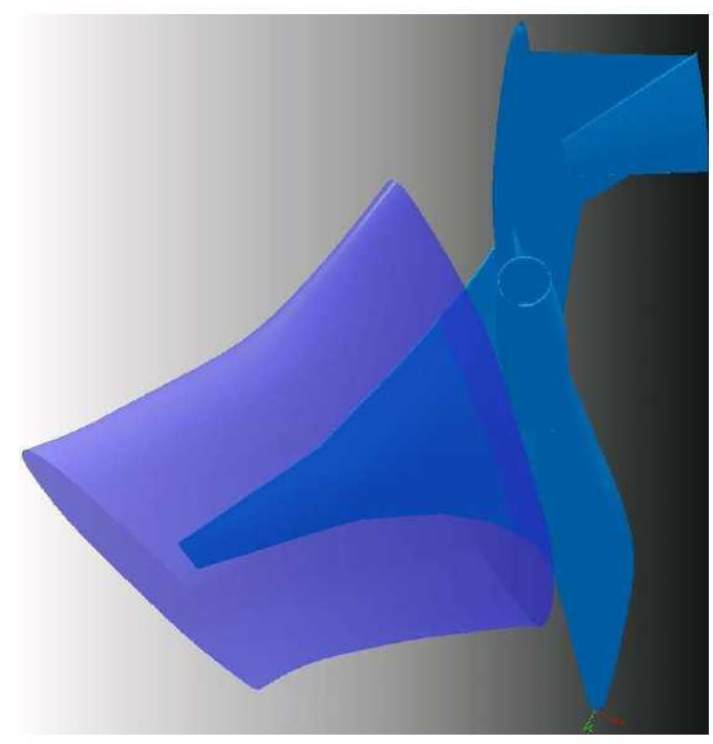

(b) Baseline ASD volume from Sculptor.

Figure 4. Sculptor control point definition.

For ease of explanation later in the paper, the movable control points that are closest to the wing root section are defined as the inboard section variables; the movable points that are closest to the wing tip, 
the outboard section variables; and the points in the middle, the mid-board section variables. Each of these three sections is defined by six control points, which creates a total of 18 movable points. To reduce the number of design variables, the points are grouped together into three groups, each representing the movable points at the three sections. Three different movements of the groups are chosen as the design variables for the optimization process. Wing twist is modified when the six points rotate about an axis that passes through a center of rotation; wing thickness is modified when the control points above the upper surface and below the lower surface move vertically in opposite directions. Finally, wing camber is modified when the movable control points above the upper surface and below the lower surface move vertically in the same direction. This creates three design variables at each of the three sections, which makes a total of nine design variables.

To carry out the optimization, bounds are placed on the design variables. These bounds are established such that the grid quality after deformation does not deteriorate to the point that the CFD solution fails to run or fails to converge to a solution. To improve the baseline mesh quality, the original VGRID ${ }^{19}$ mesh was run through a tool called CRISP. ${ }^{20}$ Different bounds of the twist design variables were tested to determine the limits beyond which the modified mesh had cells with negative volume; the variable bounds for twist were selected based on these results. The bounds for thickness and camber were chosen so that the modified designs remained close to that of the baseline. These bounds are listed in table 1 . These bounds represent the allowable movement of the control points; the actual movement of the surface is not the same. This is because the surface grid is embedded in the arbitrary shape-deformation volume and any movement in the control points causes a smooth and proportional but not identical change in the surface grid.

Table 1. Design Variable Bounds

\begin{tabular}{ccc}
\hline \hline Design variable & Lower bound & Upper bound \\
\hline Inboard twist & $-5^{\circ}$ & $5^{\circ}$ \\
Mid-board twist & $-5^{o}$ & $5^{\circ}$ \\
Outboard twist & $-5^{o}$ & $5^{o}$ \\
Inboard thickness & $-0.1 \mathrm{ft}$ & $0.1 \mathrm{ft}$ \\
Mid-board thickness & $-0.05 \mathrm{ft}$ & $0.05 \mathrm{ft}$ \\
Outboard thickness & $-0.02 \mathrm{ft}$ & $0.02 \mathrm{ft}$ \\
Inboard camber & $0.0 \mathrm{ft}$ & $0.2 \mathrm{ft}$ \\
Mid-board camber & $0.0 \mathrm{ft}$ & $0.1 \mathrm{ft}$ \\
Outboard camber & $0.0 \mathrm{ft}$ & $0.05 \mathrm{ft}$ \\
\hline \hline
\end{tabular}

Each of the CFD cases with the optimization iterations is run on 64 Intel Xeon $5130 @ 2.0 \mathrm{GHz}$ dual-core processors. The number of tetrahedral cells for each CFD mesh is approximately 3 million.

\section{Results and Discussion}

The optimization results section is divided into two subsections. The first subsection describes the optimization with just the twist variables, while the thickness and camber are kept identical to those of the baseline concept. The second subsection describes the results when all of the variables at all three wing sections are optimized.

\section{A. Twist Optimization}

The optimizer changes the twist at the three locations and is able to identify a new shape that has the same PLdB as the baseline; drag for this shape is reduced by approximately 3.32 percent with respect to the baseine. Table 2 shows the relevant metrics and design variables for both the optimum and the baseline designs. The angle of attack increases from a baseline value of $2.53^{\circ}$ to $2.8^{\circ}$. Note that a positive value for the twist indicates a drop in the leading edge of the wing section. As indicated by the optimum twist values, each of the sections is twisted leading-edge down with the mid-board section twisted as much as the upper bound will allow.

Figures 5(a) and 5(b) depict the surface pressure contours of both the baseline and the optimized configurations using three twist variables. For the sake of clarity, the nacelles are not shown with the contours on the upper surface of the wing. The lower surface of the wing has more pronounced changes 
Table 2. Results: Twist Optimization

\begin{tabular}{ccc}
\hline \hline Design variable/metric & Baseline & Optimum \\
\hline$C_{L}$ & 0.134 & 0.134 \\
$C_{D}$ & 0.01445 & 0.01397 \\
AoA $(\alpha)$ & 2.53 & 2.8 \\
PLdB & 89.3 & 89.3 \\
In-board twist & 0.0 & 3.7109 \\
Mid-board twist & 0.0 & 5 \\
Out-board twist & 0.0 & 0.8789 \\
\hline \hline
\end{tabular}

than the upper surface. Two regions of change are apparent on the lower surface. First, the high-pressure region near the leading-edge of the wing is reduced for the optimized configuration; second, as a result of the twist distribution, the trailing edge of the wing in the optimized configuration has a higher pressure compared with that of the baseline. The pressure contours on the upper surface of the optimized wing configuration are quite comparable to those of the baseline for the most part, except near the trailing edges where the shape of the contours differs from the baseline as a result of the twist distribution.

A comparison of the equivalent areas is shown in figure 6. The twist in combination with the change in the angle of attack, causes an increase in the equivalent area due to lift in the front portion and a slight decrease in the aft portion. A comparison of the ground signatures is shown in figure 7 . The maximum overpressure is reduced from the baseline; however, in the optimized configuration one of the shocks from the wing portion moves forward and increases in magnitude, while the second wing shock moves slightly aft and decreases in magnitude. Thus, the twist distribution causes a redistribution of the magnitude and location of the wing shocks, with the net result being the same perceived loudness level as the baseline.

\section{B. Full optimization}

The second optimization run was carried out with all nine design variables. In addition to the 81 runs that correspond to the orthogonal array, 71 more runs were carried out before the optimizer was unable to continue with the search step and indicated that it had reached an optimum design. The total number of CFD runs that were carried out during the optimization process was 152. Each of the CFD runs required approximately 20 minutes. However, additional analyses including Sculptor and other overheads that are associated with ModelCenter wrappers added extra time to each case; thus, each case of the optimization process required approximately 30 minutes. The total run time for the full optimization was about 3 days.

In the discussion of the results, the baseline and the optimum configurations are presented with another configuration. This configuration, termed BetterPLdB, represents an intermediate shape from the optimization process that reduces the perceived loudness below the baseline at the expense of the drag coefficient. The results of this optimization run are given in table 3. As shown in the table, the drag for the optimum was reduced 4.22 percent below the baseline drag value. The optimum configuration also maintains the same camber as the baseline perhaps because the camber of the baseline was already optimized with linear methods. Each section is twisted down, and the thickness is reduced to the lower bounds of the design variables. The results for the BetterPLdB configuration show that, unlike the optimum configuration, for boom reduction the design variables seem to alternate between positive and negative values. For example, the inboard and outboard sections are twisted in one direction, while the middle section is twisted in the opposite direction. Similarly, the thickness of the inboard and outboard sections is reduced while the mid-section thickness is increased. This perhaps is an attempt to break the wing shock to reduce the sonic boom as shown in the plots that are discussed in this section.

Figures $8(\mathrm{a})$ and $8(\mathrm{~b})$ compare the pressure contours over both the baseline and optimum configurations, over the lower and upper surfaces of the wing respectively. Once again, the lower surface contours differ appreciably compared with those of the upper surface. The contours are quite similar to the case for which only the twist variables were permitted to vary (see fig. 5(a)). In this wing shape optimization, twist plays a much larger role than the other two parameters. This conclusion is also evident based on the reduction in drag compared with that of the baseline; 3.32 percent of the reduction can be attributed to changes in the twist alone, and the remaining 0.9 percent reduction results from the reduction in thickness. 


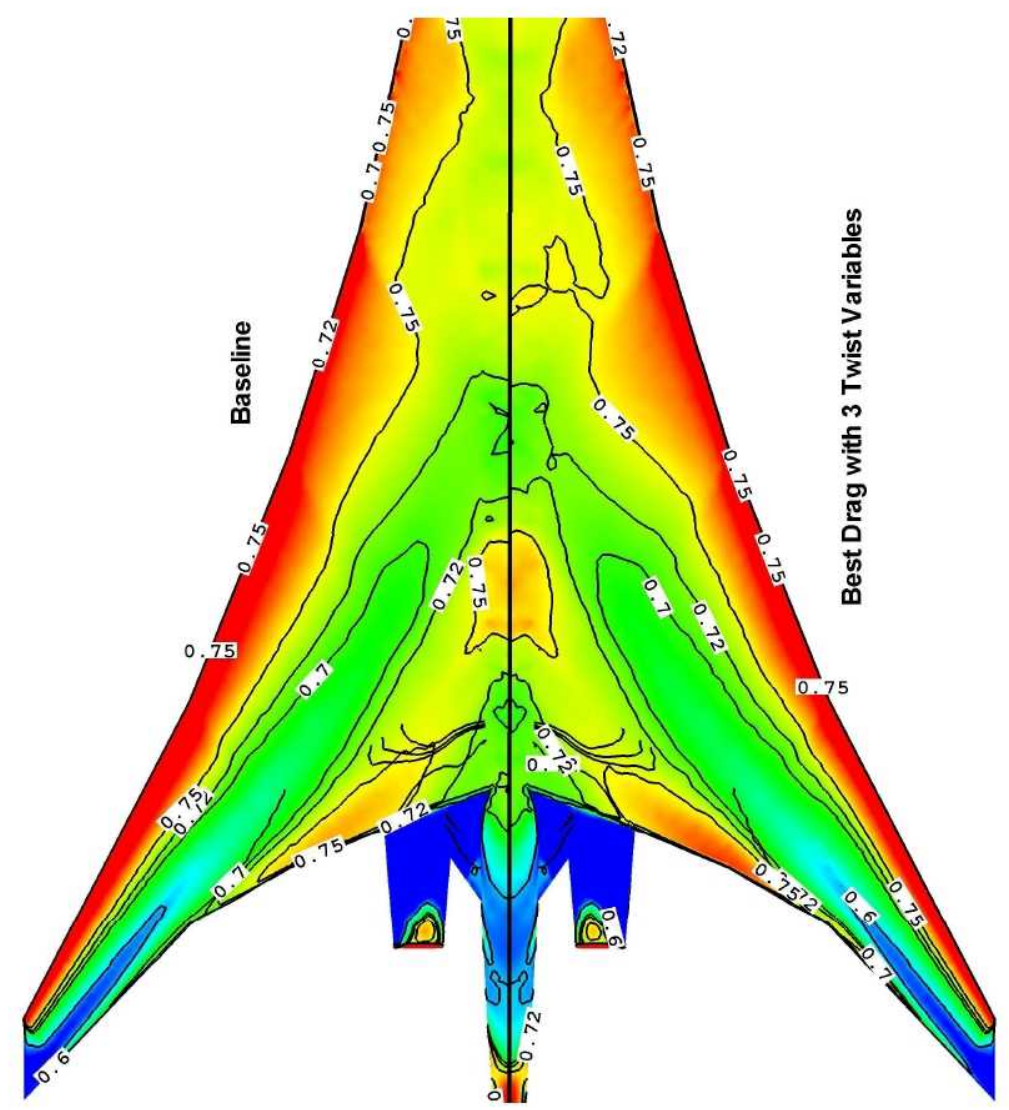

(a) Comparison of wing lower surface.

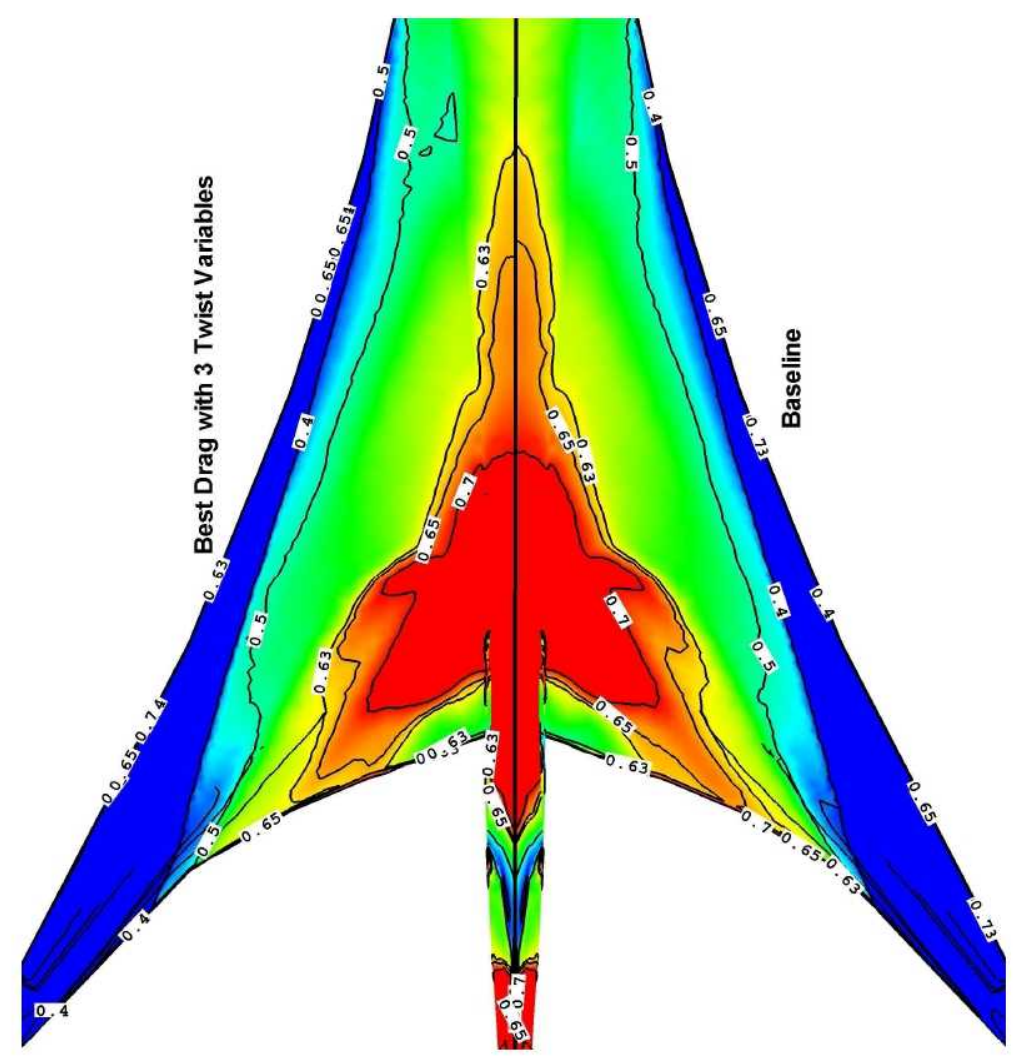

(b) Comparison of wing upper surface.

Figure 5. Pressure contour comparison over wing with only twist variables. 


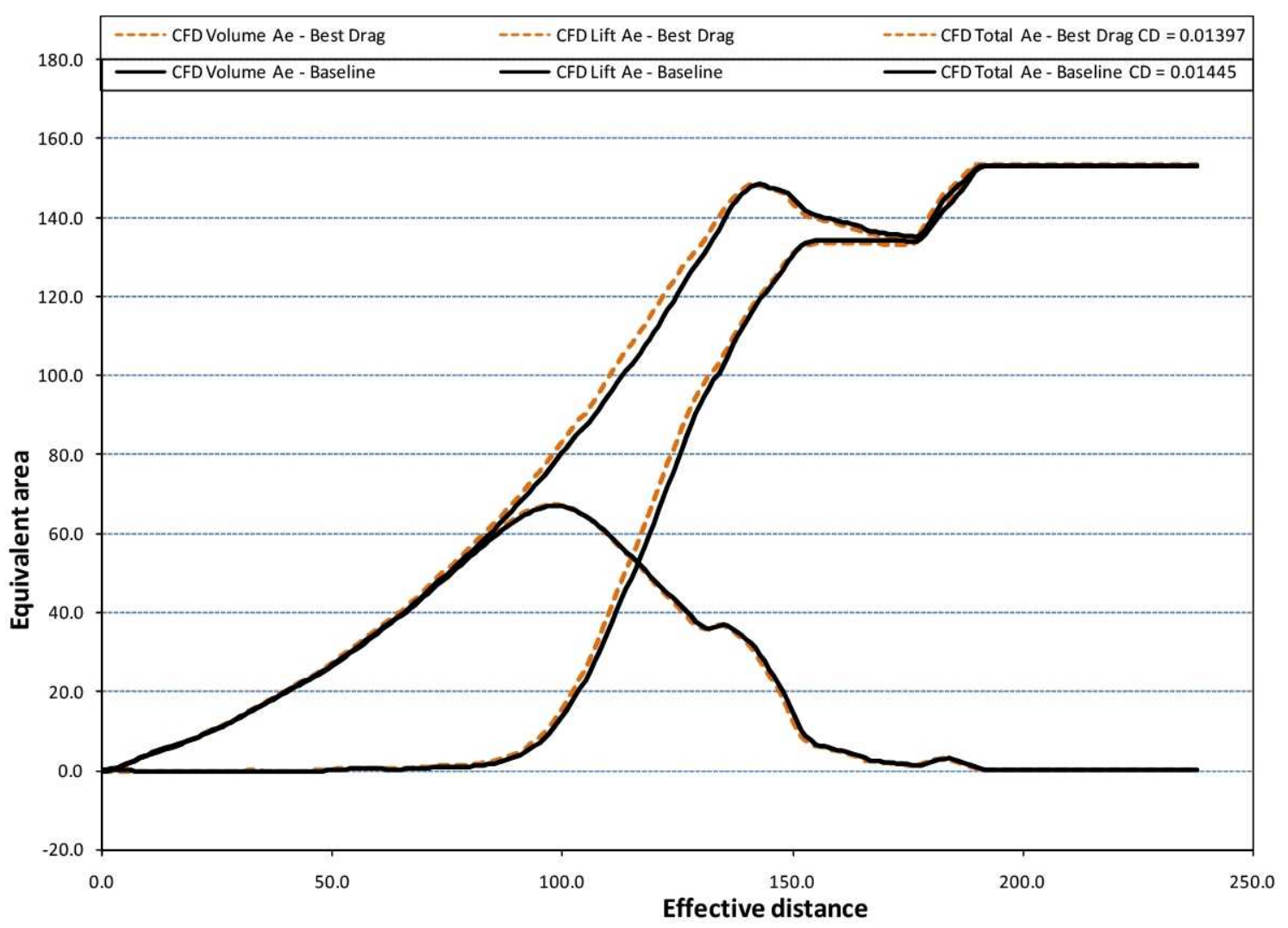

Figure 6. Comparison of equivalent areas for twist optimization

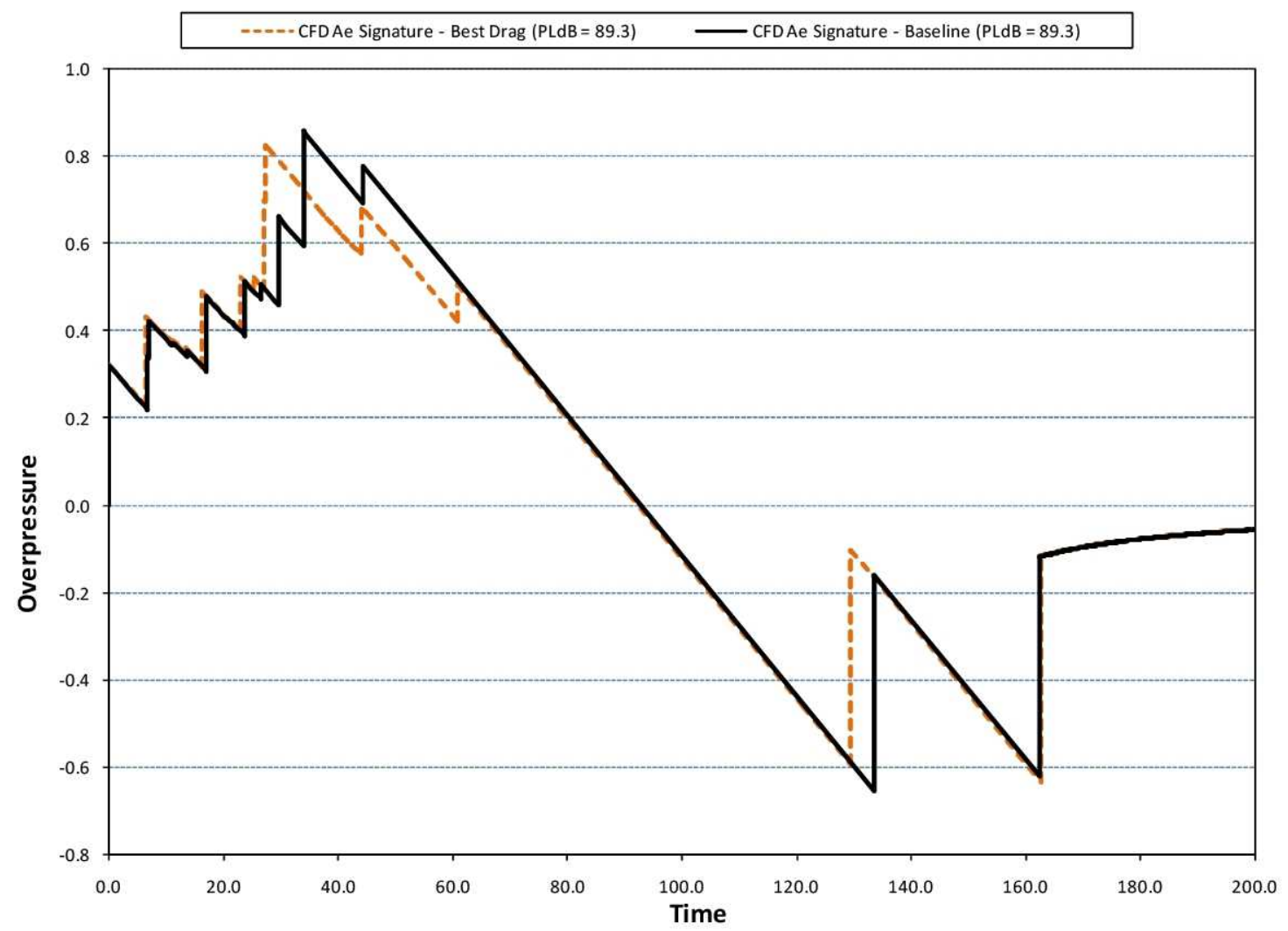

Figure 7. Comparison of ground signatures for twist optimization 


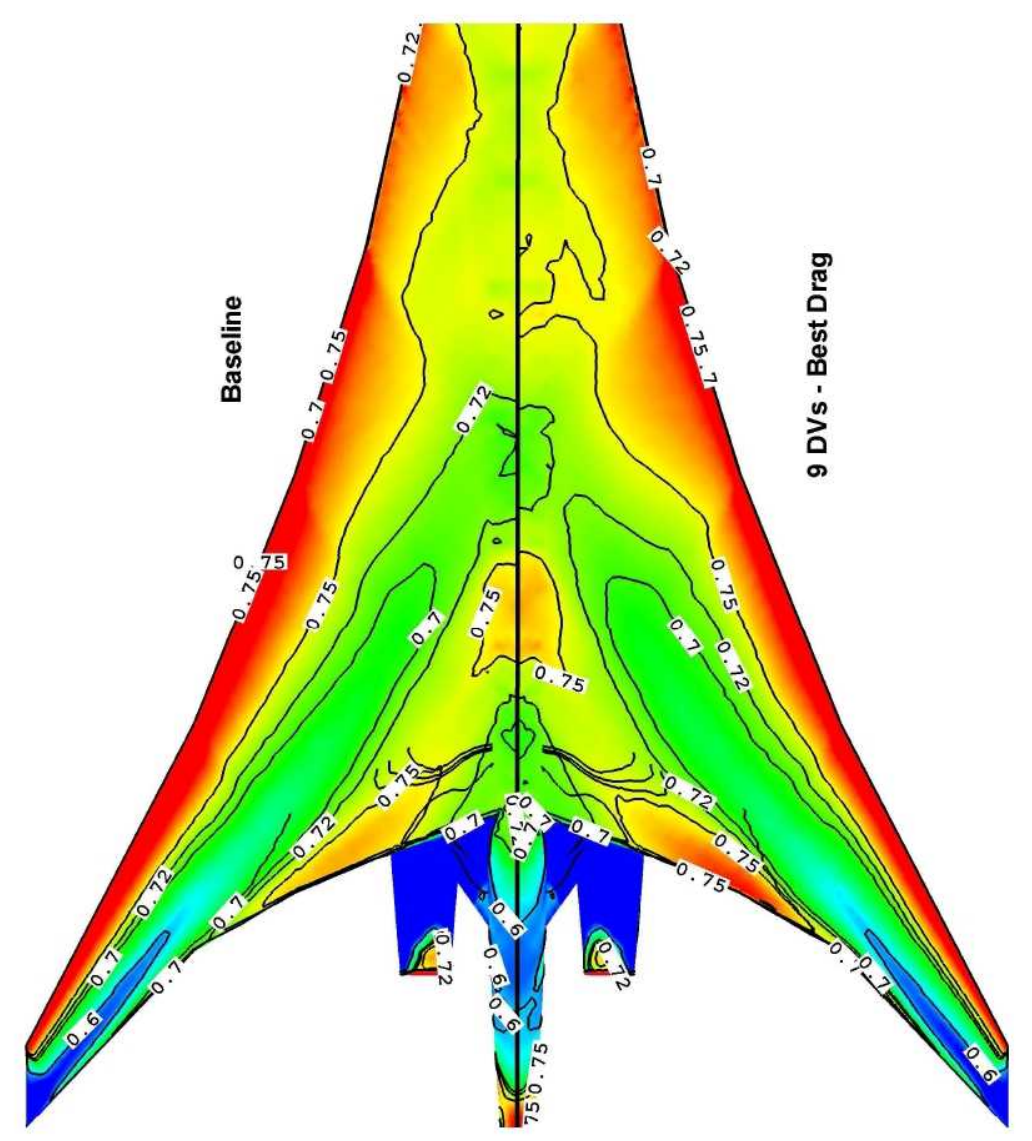

(a) Comparison of wing lower surface.

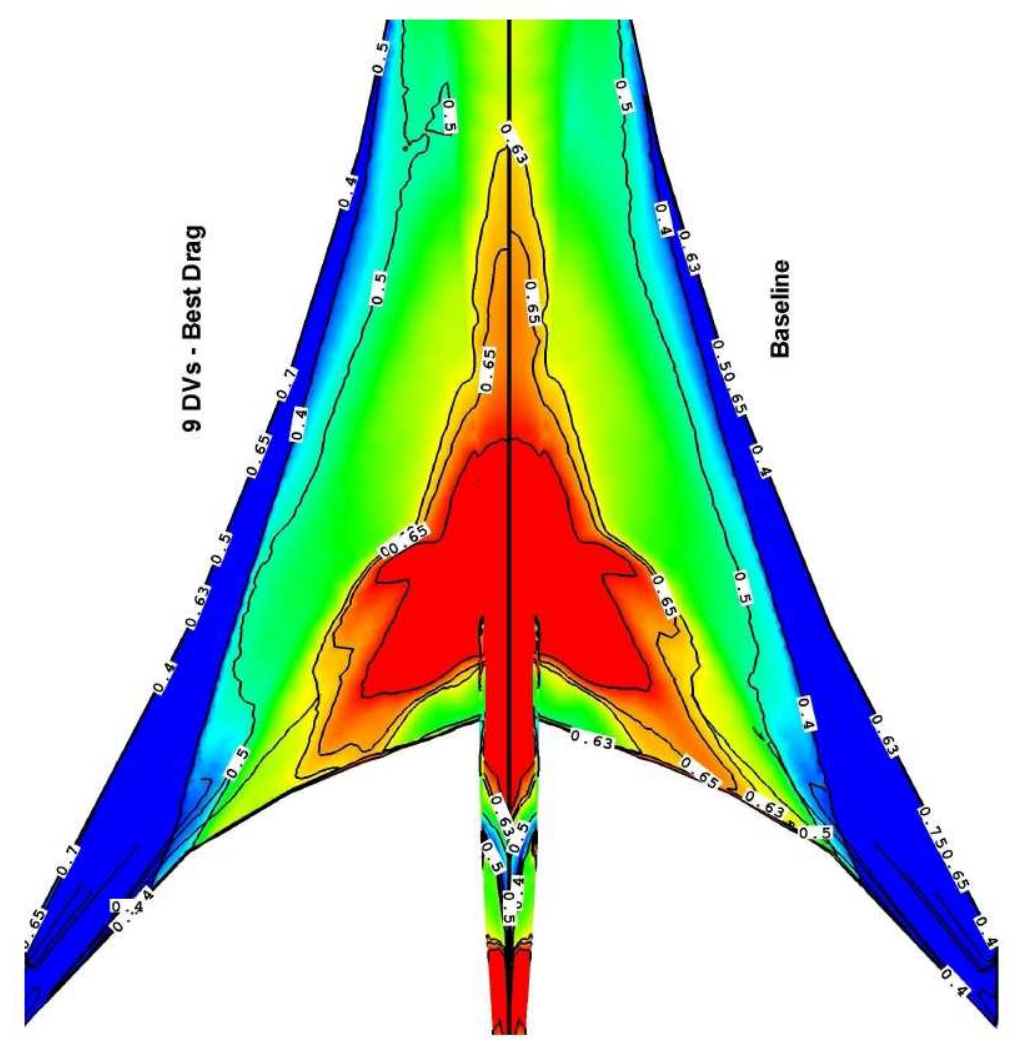

(b) Comparison of wing upper surface.

Figure 8. Pressure contour comparison over wing with all design variables. 
Table 3. Results: Nine Variable Optimization

\begin{tabular}{cccc}
\hline \hline Design variable/metric & Baseline & Optimum & Better PLdB \\
\hline$C_{L}$ & 0.134 & 0.134 & 0.134 \\
$C_{D}$ & 0.01445 & 0.01384 & 0.014566 \\
AoA $(\alpha)$ & 2.53 & 2.88 & 2.62 \\
PLdB & 89.3 & 89.7 & 88.9 \\
In-board twist & 0.0 & 5 & 4.14 \\
Mid-board twist & 0.0 & 5 & -3.92 \\
Out-board twist & 0.0 & 4.61 & 0.156 \\
In-board thickness & 0.0 & -0.1 & -0.052 \\
Mid-board thickness & 0.0 & -0.05 & 0.018 \\
Out-board thickness & 0.0 & -0.011 & -0.012 \\
In-board camber & 0.0 & 0 & 0 \\
Mid-board camber & 0.0 & 0 & 0.058 \\
Out-board camber & 0.0 & 0 & 0.03 \\
\hline \hline
\end{tabular}

Finally, figures 9(a) and 9(b) show a comparison of the pressure contours over the wing of both the baseline and the BetterPLdB configurations. In comparison with the optimum design, the BetterPLdB pressure contours are nearly the same as those of the baseline, except near the trailing edge of the wing. These differences can be explained more thoroughly with the equivalent-area plot and the computed ground signatures.

Figure 10 shows a comparison of the equivalent areas for the baseline, optimum, and BetterPLdB configurations. Because the wing is the only component that is modified, the generated equivalent areas of these configurations are fairly close to the baseline distribution. However, because the sonic boom footprint is extremely sensitive to minor changes in the equivalent area distribution, the following argument may be used to explain the comparison of the ground signatures that are shown in figure 11 . At an axial location of $100 \mathrm{ft}$, the equivalent-area corresponding to BetterPLdB design gradually rises above the baseline distribution; then at an axial location of $130 \mathrm{ft}$ it dips below the baseline distribution and then gradually rises back to the baseline values. This result is caused by the change in the sign of the adjacent twist and thickness variables. Any slope change from the baseline distribution, either positive or negative, can manifest as a shock or an expansion, respectively, in the ground signature. This is indeed the case with the ground signature of the BetterPLdB concept. On the other hand, for the optimum design, the slope of the area distribution is greater than that of the baseline at an axial location of 90 $\mathrm{ft}$; the slope remains above that of the baseline for a longer distance before it drops below the baseline as a result of reduced lift toward the trailing edge of the wing. Because of this, one of the wing shocks is pushed forward and increases in strength; the other wing shocks are significantly reduced, and the aft wing shock is reduced as well.

\section{Conclusions}

Arbitrary shape deformation has been shown to be a powerful tool to drive a CFD-based design optimization by demonstrating a viable approach for efficiently reshaping a baseline concept. The numerical optimizer relieves the design expert from performing manual CFD iterations to achieve the optimum design. The expert may analyze the final results and use engineering judgment to improve the design further. Because the basic setup has been done, future work could include optimization over multiple components with additional design variables and constraints. Note, however, that sonic boom is a global phenomenon that is dependent on and highly sensitive to the interaction between different components in addition to the changes to individual components. On the other hand, drag is a more localized phenomenon. A shape optimization study such as this one is essential after a rigorous design process has been employed to obtain a low-boom concept. As this concept is carried over into preliminary design, localized subtle changes tend to provide greater benefit in the performance metrics.

Certain difficulties are associated with the arbitrary shape-deformation design process. First, the exact deformation in the aircraft concept is not readily available because of the mapping from the control 


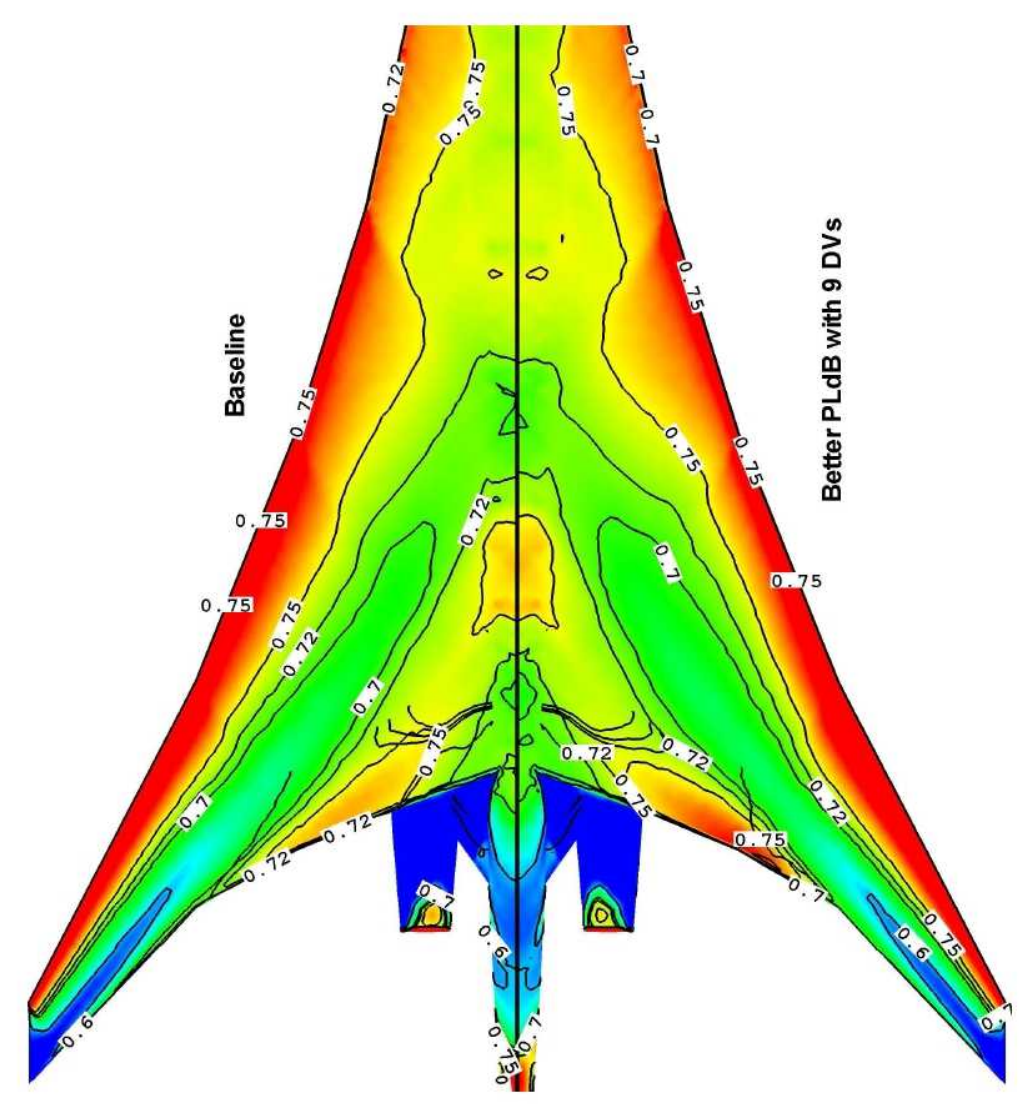

(a) Comparison of wing lower surface.

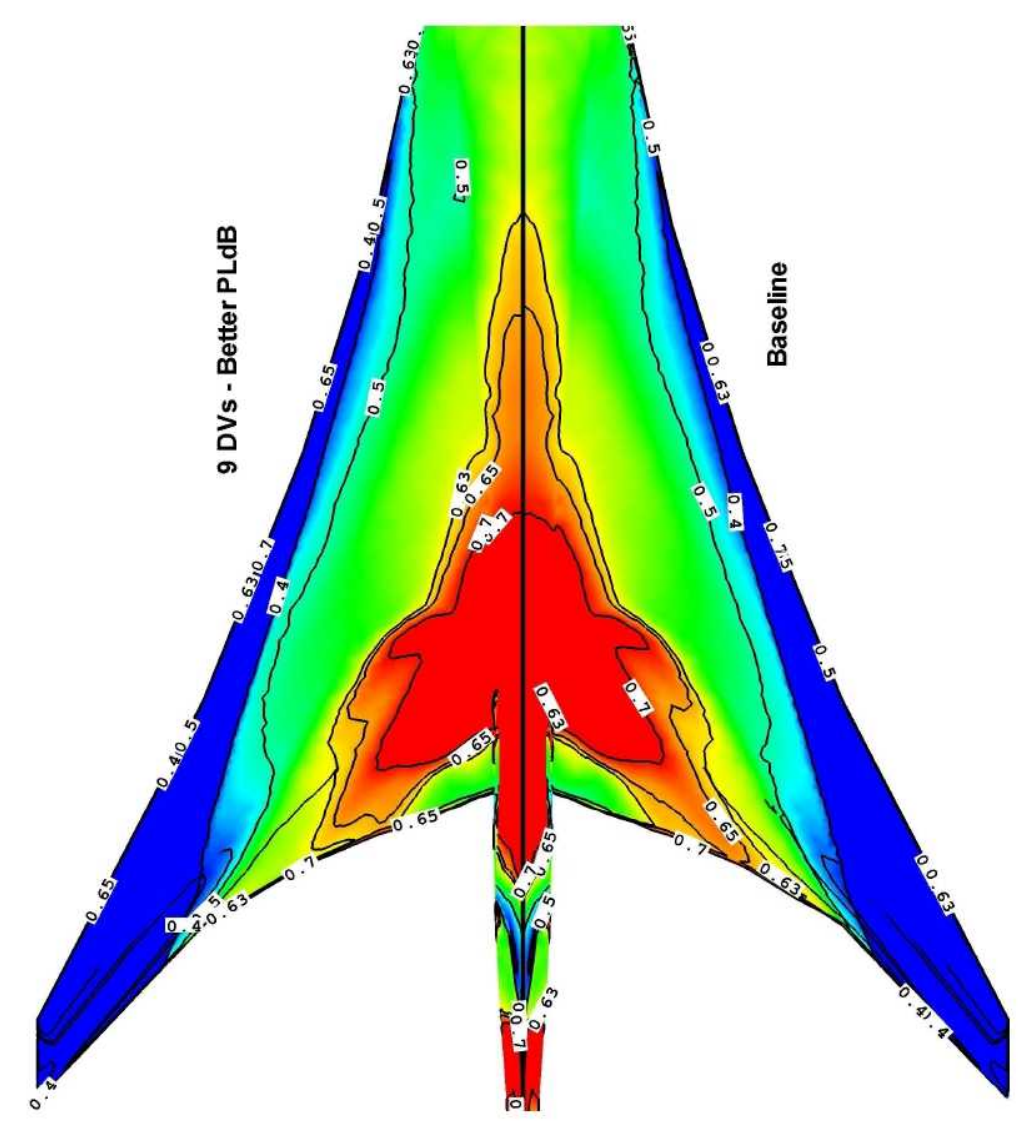

(b) Comparison of wing upper surface.

Figure 9. Pressure contour comparison over wing with all design variables. 


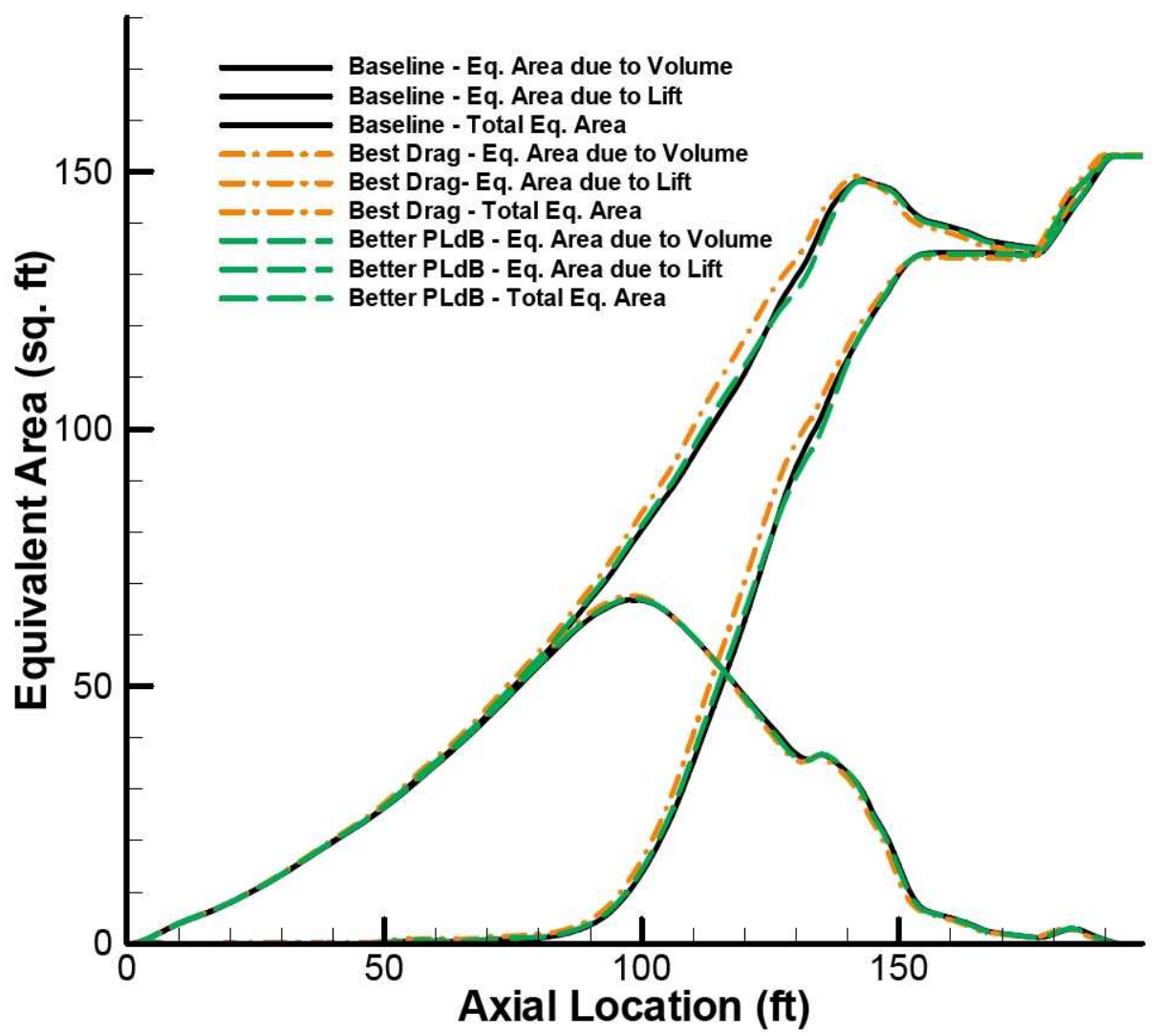

Figure 10. Comparison of equivalent areas for optimization.

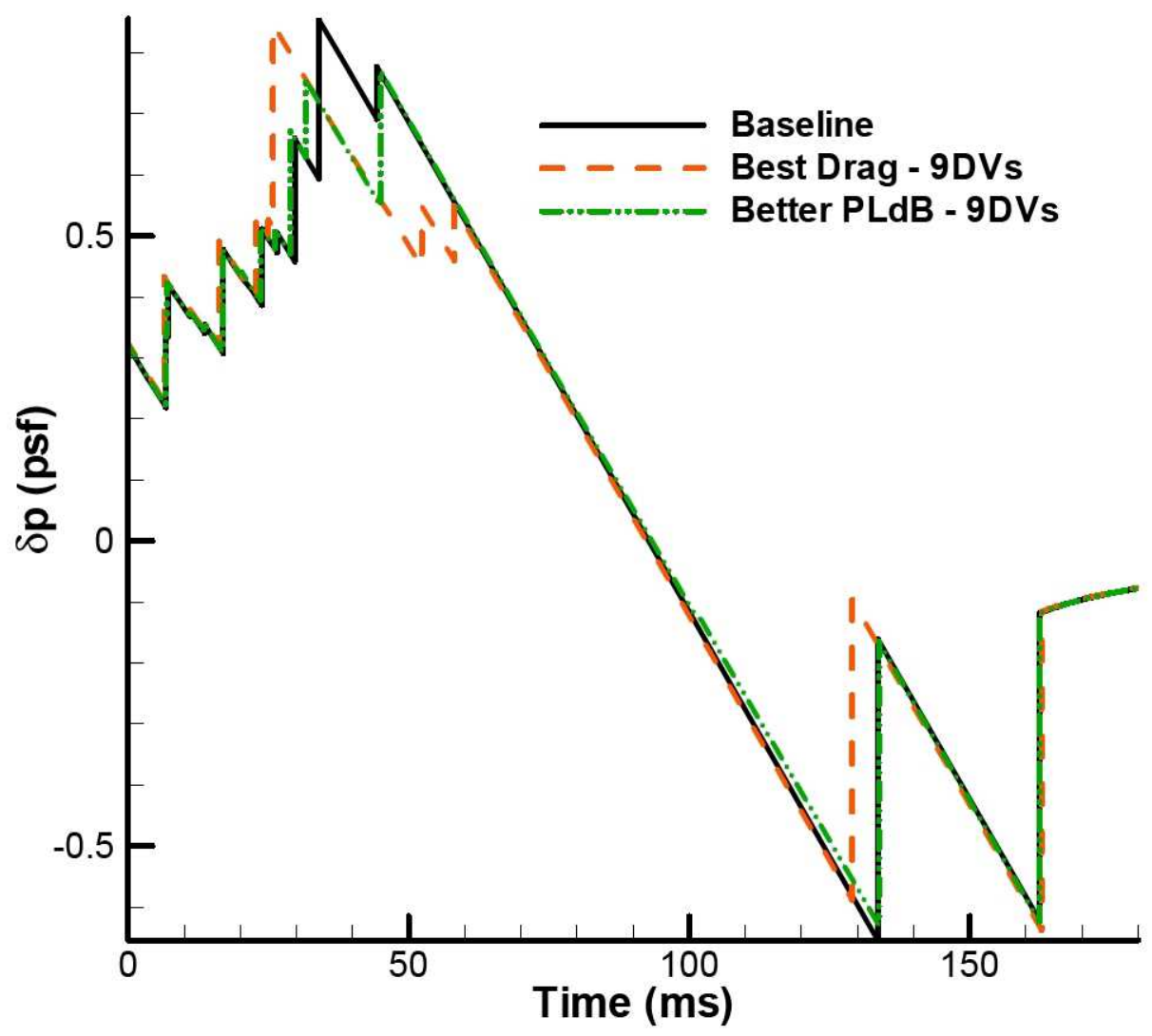

Figure 11. Comparison of ground signatures for twist optimization. 
points to the surface through the arbitrary shape-deformation volume. Second, intersection regions (e.g., wing-fuselage intersection) are much harder to control. Finally, a mesh quality improvement feature is lacking within Sulptor for shape optimization.

\section{Acknowledgments}

The work by the first author was supported in part by the NASA Project entitled "Multi-fidelity Conceptual Design Process," under NASA contract number NNL08AA00B. The authors also acknowledge the help of the engineers at Optimal Solutions, Inc., for improving the baseline mesh quality.

\section{References}

${ }^{1}$ Seebass, R., "Sonic Boom Theory," Journal of Aircraft, Vol. 6, No. 3, May 1969, pp. 177-184.

${ }^{2}$ Maglieri, D. J., Carlson, H. W., and Hubbard, H. H., "Status of Knowledge of Sonic Booms," NASA TM 80113, Hampton, VA, 1979.

${ }^{3}$ Seebass, R. and George, A., "Sonic Boom Minimization," Journal of Acoustical Society of America, Vol. 51, No. 2, 1972, pp. 686-694

${ }^{4}$ Whitham, G., "The Flow Pattern of a Supersonic Projectile," Communications on Pure and Applied Mathematics, Vol. V, 1952, pp. 301-347.

${ }^{5}$ George, A. R., "Lower Bounds for Sonic Booms in the Midfield," AIAA Journal, Vol. 7, No. 8, 1969, pp. 1542-1545.

${ }^{6}$ George, A. R. and Seebass, R., "Sonic Boom Minimization Including Both Front and Rear Shock Waves," AIAA Journal, Vol. 10, No. 10, 1969, pp. 2091-2093.

${ }^{7}$ Seebass, R. and Argrow, B., "Sonic Boom Minimization Revisited," AIAA Paper 1998-2956, June 1998.

${ }^{8}$ Plotkin, K. J., Rallabhandi, S. K., and Li, W., "Generalized Formulation and Extension of Sonic Boom Minimization Theory for Front and Aft Shaping," AIAA Paper No. 2009-1052, Jan. 2009.

${ }^{9} \mathrm{Li}$, W., Shields, E., and Le, D., "Interactive Inverse Design Optimization of Fuselage Shape for Low-Boom Supersonic Concepts," Journal of Aircraft, Vol. 45, No. 4, 2008, pp. 1381-1398.

${ }^{10} \mathrm{Li}$, W., Campbell, R., Geiselhart, K., Shields, E., Nayani, S., and Shenoy, R., "Integration of Engine, Plume and CFD Analyses in Conceptual Design of Low-Boom Supersonic Aircraft," AIAA Paper No. 2009-1171, Jan. 2009.

${ }^{11} \mathrm{Li}$, W., Shields, E., and Geiselhart, K., "A Mixed- Fidelity Approach for Design of Low- Boom Supersonic Aircraft," AIAA Paper No. 2010-0845, Jan. 2010.

${ }^{12}$ Campbell, R. L., Carter, M. B., Deere, K. A., and Waithe, K. A., "Efficient Unstructured Grid Adaptation Methods for Sonic Boom Prediction," AIAA Paper 2008-7327, Aug. 2008.

${ }^{13}$ Plotkin, K. J., "Computer Models for Sonic Boom Analysis: PCBoom4, CABoom, BooMap, CORBoom," Wyle Report WR 02-11, Arlington, VA, June 2002.

${ }^{14}$ Landon, M. et al., "SCULPTOR, Version 2.27," http://www.optimalsolutions.us, accessed November 232009.

${ }^{15}$ Frink, N. T., Pirzadeh, S., Parikh, P., Pandya, M., and Bhat, M., "The NASA Tetrahedral Unstructured Software System," The Aeronautical Journal, Vol. 104, No. 1040, 2000, pp. 491-499.

${ }^{16}$ Frink, N. T. et al., "USM3D Users Manual, Version 6.0,", http://tetruss.larc.nasa.gov/usm3d/, accessed on November 232009 .

${ }^{17}$ Cramer, E. J. and Gablonsky, J. M., "Effective Parallel Optimization of Complex Computer Simulations," AIAA Paper 2004-4461, Sept. 2004.

${ }^{18}$ Kim, H., Malone, B., and Sobieszczanski-Sobieski, J., "A Distributed, Parallel, and Collaborative Environment for Design of Complex Systems," AIAA Paper 2004-1848, April 2004.

${ }^{19}$ Pirzadeh, S., "Three-Dimensional Unstructured Viscous Grids by the Advancing-Layers Method," AIAA Journal, Vol. 34, No. 1, 1996, pp. 43-49.

${ }^{20}$ Cavallo, P. A. and Grismer, M. J., "A Parallel Adaptation Package for Three-Dimensional Mixed-Element Unstructured Meshes," Journal of Aerospace Computing, Information, and Communication, Vol. 2, No. 11, 2005, pp. 433-451. 